\title{
Pregnancy in adults with congenital heart disease
}

\author{
Elvin Zengin ${ }^{1}$, Götz Mueller ${ }^{2}$, Stefan Blankenberg ${ }^{1,3}$, Yskert von Kodolitsch ${ }^{1}$, Carsten Rickers ${ }^{2}$, \\ Christoph Sinning ${ }^{1,3}$
}

${ }^{1}$ Department of General and Interventional Cardiology, ${ }^{2}$ Department of Pediatric Cardiology, University Heart Center Hamburg, Hamburg, Germany; ${ }^{3}$ German Center of Cardiovascular Research (DZHK), Partner Site Hamburg/Kiel/Lübeck, Hamburg, Germany

Contributions: (I) Conception and design: E Zengin, C Sinning; (II) Administrative support: Y von Kodolitsch; (III) Provision of study materials or patients: E Zengin, C Sinning; (IV) Collection and assembly of data: E Zengin, C Sinning; (V) Data analysis and interpretation: All authors; (VI) Manuscript writing: All authors; (VII) Final approval of manuscript: All authors.

Correspondence to: Elvin Zengin, MD. Department of General and Interventional Cardiology, University Heart Center Hamburg, Martinistr. 52, 20246 Hamburg, Germany. Email: e.zengin@uke.de.

\begin{abstract}
Congenital heart disease (CHD) is present in $0.8-0.9 \%$ of live births. Prevalence of CHD is constantly increasing during the last decades in line with the treatment options for patients ranging from the surgical as well to the interventional spectrum. Most of the women with underlying CHD reach adulthood due to excellent surgical and interventional treatment options and most of them desire pregnancy. In Western countries, maternal heart disease is the major cause of mortality during pregnancy, thus risk estimation should be therefore individualized depending on the underlying CHD, functional class and treatment options. Pre-pregnancy counselling is mandatory in all women, especially in patients with high risk but even in patients with low risk, a plan for labor and delivery should be outlined in a heart pregnancy team. This review addresses the key element of pre-counselling, planning and successful disease management in patients with CHD during pregnancy.
\end{abstract}

Keywords: Congenital heart disease (CHD); pregnancy; management

Submitted Feb 20, 2019. Accepted for publication May 24, 2019.

doi: $10.21037 / \mathrm{cdt} .2019 .07 .01$

View this article at: http://dx.doi.org/10.21037/cdt.2019.07.01

\section{Introduction}

Congenital heart disease (CHD) is the most common form of congenital abnormalities and is present in $0.8-0.9 \%$ of all births. Most of the patients, even with complex lesions survive after birth due to surgical or interventional treatment options and reach adulthood in $95 \%$ of cases, half of them are women and most of them plan pregnancy $(1,2)$. In women with CHD pregnancy is generally well tolerated, but in contrast in up to $10 \%$ of completed pregnancies maternal cardiac complications are reported especially in those patients with complex CHD lesions (1,2). A diagnostic step of paramount importance is the use of the modified World Health Organization Risk (mWHO) scale as shown by Canobbio et al. (2) assigning a level of risk to each women regarding safety of pregnancy, in this context it has to be underlined that the safety of the mother always has priority above a successful pregnancy. Patients in class I have nearly no increased risk of maternal mortality and either none or a mild increase in morbidity. Women in class II might have a small increase in maternal mortality or a moderate increase in morbidity and those in class III have a significant increase in maternal mortality or a severe morbidity after delivery. Those in class IV, however, may carry an extremely high risk of maternal mortality or severe morbidity such that pregnancy is not advised. These patients should be counseled to avoid pregnancy. If pregnancy is confirmed in a woman in $\mathrm{mWHO}$ class IV, then termination should be discussed $(1,2)$. Although CHD is the main topic, additional complications arising during pregnancy in CHD patients have to be addressed, as heart failure and valve pathologies are often encountered together with rhythm disorders in 
these patients (1-3).

A novel aspect which is outlined in the guidelines is the pregnancy heart team with the minimum staff of a cardiologist, obstetrician and anesthesiologist; however additional specialties are often needed like the cardiothoracic surgeon and other medical specialties $(1,2)$. The team discusses the pre-counselling, management and delivery of the patient in the context of the $\mathrm{mWHO}$ risk class and files the conclusions and decisions which have to be available to the patients and treating physician in 24 hours $(1,2)$.

The review focusses on the most common heart defects and problems related to these heart defects. New aspects from the current guidelines will be discussed as well. In addition a short summary of the most important hemodynamic changes during pregnancy will be outlined in this review manuscript.

\section{Methods}

\section{Literature search}

Until December 2018 a literature search was performed in PubMed. The following combinations of keywords were used: pregnancy and CHD, morbidity and mortality in pregnancy, guidelines cardiovascular disease and pregnancy. These search terms had to be identified anywhere in the text in the articles. Both qualitative and quantitative studies were considered to elucidate the use of the different aspects regarding CHD, pregnancy and combination of both. The search was restricted to original research, humans, and papers published in English at any date. All abstracts were reviewed to assess whether the article met the inclusion criteria. The key inclusion criterion was the presence of any cardiovascular disease in combination with pregnancy. Most of the suggestions depend on the summary statement of the American Heart Association (2) and the guidelines of the European Society of Cardiology (1). After this selection process, manual search of the reference lists of all eligible articles was performed. Two authors (i.e., E.Z. and C.S.) assessed independently the methodological quality of the qualitative and quantitative studies prior to their inclusion in the review.

\section{Hemodynamic changes during pregnancy}

Pregnancy induces alterations in organ systems to meet the demands of both mother and fetus during pregnancy $(1,2)$. Thus these changes can be challenging or even dangerous for women with CHD as the cardiovascular system might be too impaired to fulfill these normally well tolerated changes.

Cardiac output increases during a singleton pregnancy between $30-50 \%$ with additional $10-20 \%$ in a twin pregnancy, a peak increase occurs around the $24^{\text {th }}$ week after conception. These changes in cardiac output are happening along with an increase in blood volume with approximately $40 \%$ increase in a singleton pregnancy and around $70 \%$ in twin pregnancies with a plateau at the $32^{\text {nd }}$ week of pregnancy $(1,2)$. In addition to the changes in cardiac output and blood volume, the heart rate increases by 10 to 20 beats per minute reaching its maximum in the late second trimester or first part of the last trimester. To allow these changes to develop pulmonary vascular resistance (PVRi) and systemic vascular resistance drop considerably early during pregnancy and systemic vascular resistance rises again after the $24^{\text {th }}$ week after conception to reach pre pregnancy levels before term $(1,2)$.

Inappropriate rising levels of $\mathrm{N}$-terminal pro $\mathrm{B}$-type natriuretic peptide (NT-proBNP) during pregnancy indicate malalignments of volume and pressure adaptations in the cardiovascular system. Median NT-proBNP levels during pregnancy, however, are twice the level compared to non-pregnant controls. Recent guidelines have pointed at the potential use of NT-proBNP as biomarker of risk during pregnancy with a negative predictive value of approximately $95 \%$ and with a level of $128 \mathrm{pg} / \mathrm{mL}$ at the $20^{\text {th }}$ week of pregnancy $(1,2)$ but its role has to be further determined in future studies.

The increase of cardiac output peripartum is important to highlight, as it rises from $10 \%$ in the first stage of labor to around $30 \%$ during the second stage, leading to additional hemodynamic challenges which are ameliorated by effective epidural anesthesia (2). Postpartum, the major hemodynamic challenges are an immediate rise of cardiac output and an increase of blood pressure in the first two days after delivery which are returning back to normal levels in the following weeks.

\section{Preconception counselling}

The pre-conception evaluation is of paramount importance and has to assess the individuals risk. It should as well outline strategies and possible treatment options reducing the risk during pregnancy. Although it is important for the patient to outline the risk on the one hand, it has to be pointed out that most patients can complete pregnancy 
with a low-risk of mortality or morbidity on the other hand $(1,2)$. For adequate risk estimation a minimum of examinations has to be scheduled with electrocardiogram, echocardiography, exercise test and a blood draw. The concept of risk in the manuscript is described as any complication during pregnancy affecting the mother or the fetus which may result in any harm to either or both. An example of preconception counseling are diseases resulting in pathologies of the aorta like bicuspid aortic valve or coarctation of the aorta (4). If for example a pathology of the aorta is known, a magnetic resonance imaging (MRI) or if not feasible computer tomography (CT) scan of the aorta has to be done before pregnancy $(5-7)$ to evaluate if the pathology has to be corrected before pregnancy.

\section{Possible complications of pregnant women with CHD}

\section{Arrhythmia risk during pregnancy}

Pregnancy is related to physiologic changes, in particular the increase in heart rate as well as a shortening of the specific cardiac time intervals. Often a left-ward shift of the ECG axis due to elevation of the diaphragm during pregnancy is recognized (2). It is not uncommon to observe an increase in ectopic beats which are usually without a pathologic substrate. However, during pregnancy in patients with CHD, the threshold for ventricular and supraventricular arrhythmias are also lowered and therefore, might be frequently encountered during pregnancy $(1,2)$.

The principles of management during pregnancy in general follow the recommendations which are presented in the respective guidelines $(8,9)$. The most common aspects, which have to be considered is the influence of arrhythmia in the context of hemodynamic tolerance in the described congenital heart defect, in addition, if the medication used can cross the placental border and could affect the fetus $(1,2)$. In the case of paroxysmal supraventricular tachycardia involving the atrioventricular node in a reentrant circuit, adenosine is the medication of choice as it is not crossing the placental border. Alternatively, beta-blockers can be used to achieve frequency control. For patients with atrial fibrillation or atrial flutter, beta-blocking agents are recommended as first-choice with sotalol being an option to achieve conversion in patients with atrial flutter, atrial fibrillation or even ventricular tachycardia $(1,2)$. For patients with ventricular tachycardia in their previous medical history, beta-blocker therapy should be given throughout the whole pregnancy. In acute stable situations, intravenous lidocaine should be considered as well as direct current cardioversion $(1,2)$.

\section{Mechanical heart valves and anticoagulation}

The presence of mechanical heart valves and the need of anticoagulation are risks for the mother as well as the fetus and/or newborn as it imposes additional threats for the patient. For the mother the most dangerous situation is thromboembolism and/or thrombotic complications of the mechanical valve resulting in stroke and/or cardiogenic shock needing emergency cardiac surgery or lysis therapy $(1,2)$. The risk of complications is especially increased if there are older types of heart valves, like the early cage or ball mechanical heart valves and in particular if there is more than one mechanical heart valve. Complications are elevated when the function of the mechanical valve is impaired before pregnancy and if heparin is used for anticoagulation during the whole pregnancy. From the different anticoagulation schemes warfarin/phenprocoumon is the safest as long as intake is lower than $5 \mathrm{mg}$ per day and if the substances are not taken in the first 6-12 weeks during organogenesis of the fetus. In this time low-molecular weight heparin with strict anti-Xa monitoring (target level: $0.8-1.2 \mathrm{U} / \mathrm{mL}$ ) is the medication of choice. Lowdose aspirin with $100 \mathrm{mg}$ per day should be considered in addition to the anticoagulant for patients with a mechanical heart valve. Switch to intravenous heparin is recommended with a mechanical heart valve one week before delivery in hospital setting. Caesarean section should be offered to the patient to avoid complications during labor $(1,2)$.

\section{Heart failure during pregnancy}

For patients with CHD hemodynamic changes during pregnancy, arrhythmias and discontinuation of certain heart failure medications can result in acute or worsening chronic heart failure $(10,11)$. The clinical outcome depends on the type of heart failure the patient develops. Patients with transposition of the great arteries (TGA) and Senning or Mustard surgery, congenital corrected TGA (ccTGA), Eisenmenger syndrome or impaired ventricular function with an $\mathrm{EF}$ below $40 \%$ are prone to develop complications and pulmonary edema. Furthermore, patients after correction of Fallot Tetralogy or pulmonary atresia can show symptoms of right-sided heart failure $(1,2,10,11)$. The use of NT-proBNP as biomarker might be useful in this context. It is mandatory 
to counsel this cohort of patients before and during pregnancy in a pregnancy heart team, outline a delivery plan and refer patients to tertiary care centers.

\section{Specific congenital heart defects and considerations for planning pregnancy and delivery}

\section{Pulmonary arterial bypertension and Eisenmenger syndrome ( $\mathrm{mWHO}$ class III to IV)}

Pulmonary arterial hypertension is characterized by a mean pulmonary artery pressure of $\geq 25 \mathrm{mmHg}$, in addition LV filling pressure of $\leq 15 \mathrm{mmHg}$ as assessed by right heart catheter in wedge position and PVRi of $>3$ Wood units (WU) (12). Before addressing the anatomical problem with an operation however, hemodynamic aims have to be met which are summarized in the current guidelines with a PVRi indexed of $\leq 8 \mathrm{WU}(13,14)$ per square meter of body surface area in right heart catheterization to avoid right heart failure after surgery. In this setting especially women with PAH develop clinical symptoms during pregnancy regardless of the underlying congenital heart defect.

For the spectrum of pulmonary hypertension in general the outcome of the mother has improved although in idiopathic pulmonary arterial hypertension maternal mortality remains high with about $16-30 \%$ mortality in all pregnant patients $(1,12,15)$. This is mainly due to pulmonary hypertensive crisis, pulmonary thrombosis and/ or right heart failure. In line with the increased maternal risk, the risk for the fetus is as well increased due to often preterm delivery, reduced cardiac output of the mother and hypoxemia (1). In patients with $\mathrm{PAH}$, management should follow the current guidelines for pulmonary arterial hypertension regarding diagnosis and treatment whereby echocardiography is the key imaging modality (12). Pregnancy in PAH patients is a high-risk situation and patients should be treated as suggested in the current PAH guidelines. However endothelin-receptor antagonists should be discontinued during pregnancy, instead the use of sildenafil is recommend $(1,12)$. The mode of delivery has to be determined in the pregnancy heart team and should be appointed in a tertiary care center offering the needed facilities for intensive care medicine, obstetrics and anesthesia. During delivery regional anesthesia is usually favored over general anesthesia. Fluid balance and optimization of right ventricular function are important determinants of a good outcome. Patients remain at high risk for many months post-delivery, therefore individualized counselling is of importance to discuss the need for ongoing therapies and the avoidance of future pregnancies $(1,2,12)$.

\section{PAH and Eisenmenger's syndrome (mWHO IV)}

Patients with Eisenmenger's syndrome have even an increased risk of adverse events during pregnancy because of the increased cyanosis, right-to-left shunting and thus the risk of paradoxical systemic embolism. During pregnancy systemic vascular resistance drops and right-to-left shunt can increase, thus may worsening cyanosis and decreasing cardiac output. These factors result in a high maternal mortality with $20-50 \%$-however even termination of the pregnancy is accompanied with adverse events. Thus, preconception counselling and contraception in these patients is of paramount importance $(1,2,12)$.

\section{Atrial septum defect, ventricular septum defect and atrioventricular septal defect ( $m W H O$ class I or II up to III in AVSD)}

In patients with small or repaired atrial or ventricular septal defects the risk of complications is low. Sometimes in both diseases thromboembolism or arrhythmia may complicate pregnancy, however in otherwise asymptomatic patients pregnancy is well tolerated. Risk can be elevated in patients with AVSD, especially in patients with complete AVSD due to the increased risk of developing heart failure or valvular regurgitation. In these patients follow-up is recommended at least once in each trimester $(1,2)$.

\section{Aortic stenosis and left ventricular outflow tract obstruction ( $m W H O$ class II to III)}

In women of childbearing age most commonly bicuspid aortic valve is the cause for moderate to severe aortic valve stenosis, patients with severe stenosis of the aortic valve may become symptomatic during pregnancy $(1,2)$. For diagnosis during pregnancy echocardiography is the method of choice and further to determine presence of aortic stenosis and define its severity. As additional test in patients without symptoms exercise testing should be done with bicycle stress to screen for arrhythmias, pathologic blood pressure response, reduced exercise capacity and changes in the ECG $(2,16)$. Most commonly pregnancy is well tolerated in asymptomatic patients with mild (mean gradient $<25 \mathrm{mmHg}$ ) or moderate (mean gradient, $25-40 \mathrm{mmHg}$ ) 
aortic stenosis. Patients with severe valve stenosis (mean gradient $>40 \mathrm{mmHg}$, valve opening area $<1.0 \mathrm{~cm}^{2}$ ) may tolerate pregnancy well and remain asymptomatic, but careful individualized assessment is needed $(2,17,18)$. For management it is essential to recognize that these patients can become symptomatic during pregnancy due the fact that volume load increases, resulting in a higher gradient. At the beginning of the pregnancy an ECG should be reviewed regarding signs of cardiac hypertrophy or ischemia for risk stratification (2). In patients with non-severe aortic stenosis in general conventional delivery is recommended, in patients with symptomatic or severe stenosis induction is recommended at least at the beginning of the $39^{\text {th }}$ week of gestation. A detailed delivery plan discussed in the heart pregnancy team is mandatory $(1,2)$. After delivery asymptomatic patients should be monitored like the general obstetric patient, furthermore telemonitoring with ECG for 24 hours should be scheduled. Left ventricular outflow tract obstruction is handled the same way, however balloon dilatation is not a treatment option to consider in these patients.

\section{Dextro-TGA (D-TGA) (mWHO class III to IV if systemic ventricular function is impaired)}

The clinical outcome of mother and child in pregnant patient with D-TGA depends on which surgical procedure was done during the childhood of the mother, which was mainly the palliative atrial switch surgery up to the late 1980s or anatomical correction in arterial switch procedure afterwards (3).

\section{Patients after atrial switch procedure}

The atrial repair usually refers to the technique by Senning or Mustard to redirect the blood flow on the atrial level either using autologous material (Senning) or Dacron (Mustard). However, although the blood flow is according to the normal blood flow, the right ventricle functions as systemic right ventricle $(1,2)$. Following the surgery patients after atrial switch procedure are at a high risk of developing sinus node dysfunction or atrial arrhythmias resulting from scarring due to the original reparative surgery. Further, these patients have a high-risk of ventricular arrhythmias leading to sudden cardiac arrest $(3,19,20)$. Atrial switch operation may also lead to baffle leak or obstruction, heart failure due to dysfunction of the systemic right ventricle or pulmonary hypertension $(21,22)$. In the context of the systemic right ventricle physiology pregnancy might be poorly tolerated in this cohort of patients. The increased blood volume, heart rate, stroke volume, and cardiac output that occur in pregnancy may unmask heart failure, worsen tricuspid regurgitation or exacerbate arrhythmias $(2,23)$. In this context some data suggest that pregnancy in patients with atrial repair will be subject to increased decline of right ventricular function and/or atrioventricular valve regurgitation. This change even might be irreversible after pregnancy $(2,23)$. In patients desiring to become pregnant this issue has to be discussed in the pre-counselling visit. The diagnostic procedures before pregnancy should include echocardiography or MRI, exercise testing and a 24-hour Holter ECG. The use of natriuretic peptides is suggested in addition to identify patients early with ventricular pressure and/or volume overload. Patients with moderate to severe impairment of ventricular function should be advised against pregnancy $(1,2)$. Patients should be examined every 4-6 weeks in the second trimester and 2-4 weeks in the third trimester with an individualized scheme $(1,2)$. Delivery is depending on the functional status of the mother, however patients with symptoms of heart failure might need monitoring or even caesarian delivery to avoid additional strain and stress during delivery $(1,2)$. In general patients without hemodynamic compromise and a good functional status tolerate vaginal delivery with a facilitated second stage using regional anesthesia. Caution is warranted during the first 24 to 48 hours after delivery because the shifting in blood volume might result in symptoms of heart failure even in patients without previous symptoms.

\section{Patients after arterial switch procedure}

The modern surgical anatomical correction of D-TGA is the arterial switch procedure $(1,2)$. However this operation was not commonly done until the late 1980s and early 1990s so long-term experience is missing with the outcome after using this surgical technique. More severe complications like aortic dilatation, aortic valve regurgitation, pulmonary artery stenosis or coronary abnormalities were only reported occasionally $(24,25)$. Thus the diagnostic algorithm and management during pregnancy will be dictated by symptoms or residual hemodynamic abnormalities. In asymptomatic woman with arterial switch and no hemodynamic abnormalities, evaluation and management may be similar to that in lower-risk cardiac patients $(2,26)$.

\section{ccTGA (mWHO class III)}

In patients with ccTGA in addition to the TGA the ventricles 
are as well switched with a physiologic normal blood flow, however the right ventricle functions as systemic ventricle $(1,2)$. In these patients the risk depends on status of the systemic right ventricle, presence of arrhythmias and valvular regurgitation as surrogate of right heart failure. Management can be complicated due to presence of associated lesions like ventricular septum defect or pulmonary valve stenosis. In these patients close monitoring of right heart function with echocardiography is recommended every $4-8$ weeks together with symptoms to optimize treatment $(1,2)$.

\section{Tetralogy of Fallot (ToF) (mWHO class II)}

The ToF is the most common type of cyanotic CHD and nowadays almost all patients underwent correction procedure in childhood and reach reproductive age in industrialized countries. In general these patients tolerate pregnancy well, however patients taking cardiac medications and having structural abnormalities and/or arrhythmia have an increased risk of complications like developing heart failure during pregnancy. Usually these pregnant women should undergo a follow-up visit each trimester and in case of severe pulmonary regurgitation a monthly visit of the mother should be scheduled (1). If right ventricular failure occurs during pregnancy, treatment with diuretics should be started and bed rest advised. Early delivery or, rarely, transcatheter valve implantation could be considered in those who do not respond to conservative treatment.

\section{Patients with single-ventricle physiology/Fontan-palliation ( $m W H O$ class III to IV)}

The term single-ventricle physiology commonly refers to the presence of a dominant ventricle, either the anatomic left or right ventricle and the presence of a hypoplastic accompanying ventricle. Although there are a lot of cardiac defects leading to a functional single-ventricle physiology the common feature is the need of the single-ventricle to circulate the blood of the whole body $(1-3,26)$. There are a variety of surgical palliation procedures, the most definite one was described by Fontan redirecting the deoxygenated systemic venous blood to the pulmonary arteries without an intervening ventricle functioning as pump $(1-3,26)$. Thus the main sequelae after Fontan procedure are an increased systemic venous pressure, reduced cardiac output and cardiac reserve (27). Most commonly the patients are doing well after the Fontan procedure but over time arrhythmias, ventricular function impairment, portal hypertension and protein-losing enteropathy $(28,29)$ may occur. For delivery itself certain pathophysiological changes have to be considered: the variable increase in central venous pressure, decrease in cardiac output, the vasoplegic effects of anesthetic agents and fluid shifts noted in the postpartum period, may all result in volume overload with increased demand for diuretics $(1,2)$. During pregnancy patients are at a high risk of thromboembolic events due to the modified anatomy following Fontan palliation. In fact regarding the high rate of obstetric complications like miscarriage, early rupture of membranes and early start of labor with smallfor-gestational age children, all patients need a thorough evaluation before pregnancy. At affirmation of pregnancy repeat echocardiogram should be considered at the start of each trimester to determine ventricular function. For patients with a history of arrhythmias or clinical symptoms of palpitations, ambulatory ECG is indicated. Baseline arterial oxygen saturation and liver function tests should be obtained. Oxygen saturations should be measured at each clinic visit to detect a decreasing functional status of the patient early $(1,2)$. As outlined for patients with systemic right ventricle, vaginal delivery should be recommended with a facilitated second stage of labor and as alternative for patients with a poor status due to heart failure and congestion caesarean delivery should be advised $(1,2)$. These patients as well have an increase of blood volume during the first 24 to 48 hours resulting in optional congestion which should then be treated with additional diuretic therapy. However cardiac monitoring should be done in the first 24 hours after delivery and if the patient has hemodynamic compromise the patient should be admitted to the intensive cardiac care unit for 24 to 48 hours $(1,2)$.

\section{Summary}

As a result of the improved medical and surgical therapy more patients with CHD reach adulthood and often have the wish for family planning and child bearing. Even in patients with complex heart disease this wish can often be fulfilled with the support of expert consulting. Of paramount importance is the fact that each patient has to be counselled by a heart pregnancy team regarding the risk for the patient and child. Depending on the underlying cardiac diagnosis individualized risk estimation should be done before pregnancy, especially women with mWHO class II-III and IV with an increased peri- and postpartum risk of events. Pre-pregnancy counselling as well as management during pregnancy and labor should be discussed in expert centers. 


\section{Acknowledgments}

None.

\section{Footnote}

Conflicts of Interest: The authors have no conflicts of interest to declare.

Ethical Statement: The authors are accountable for all aspects of the work in ensuring that questions related to the accuracy or integrity of any part of the work are appropriately investigated and resolved.

\section{References}

1. Regitz-Zagrosek V, Roos-Hesselink JW, Bauersachs J, et al. 2018 ESC Guidelines for the management of cardiovascular diseases during pregnancy. Eur Heart J 2018;39:3165-241.

2. Canobbio MM, Warnes CA, Aboulhosn J, et al. Management of Pregnancy in Patients With Complex Congenital Heart Disease: A Scientific Statement for Healthcare Professionals From the American Heart Association. Circulation 2017;135:e50-87.

3. Baumgartner H, Bonhoeffer P, De Groot NM, et al. ESC Guidelines for the management of grown-up congenital heart disease (new version 2010). Eur Heart J 2010;31:2915-57.

4. Sinning C, Zengin E, Kozlik-Feldmann R, et al. Bicuspid aortic valve and aortic coarctation in congenital heart disease-important aspects for treatment with focus on aortic vasculopathy. Cardiovasc Diagn Ther 2018;8:780-8.

5. Roos-Hesselink JW, Budts W, Walker F, et al. Organisation of care for pregnancy in patients with congenital heart disease. Heart 2017;103:1854-9.

6. Greutmann M, Pieper PG. Pregnancy in women with congenital heart disease. Eur Heart J 2015;36:2491-9.

7. Ohuchi H, Tanabe Y, Kamiya C, et al. Cardiopulmonary variables during exercise predict pregnancy outcome in women with congenital heart disease. Circ J 2013;77:470-6.

8. Priori SG, Blomstrom-Lundqvist C, Mazzanti A, et al. 2015 ESC Guidelines for the management of patients with ventricular arrhythmias and the prevention of sudden cardiac death: The Task Force for the Management of Patients with Ventricular Arrhythmias and the Prevention of Sudden Cardiac Death of the European Society of Cardiology (ESC). Endorsed by: Association for European
Paediatric and Congenital Cardiology (AEPC). Eur Heart J 2015;36:2793-867.

9. Kirchhof P, Benussi S, Kotecha D, et al. 2016 ESC Guidelines for the management of atrial fibrillation developed in collaboration with EACTS. Eur Heart J 2016;37:2893-962.

10. Roos-Hesselink J, Baris L, Johnson M, et al. Pregnancy outcomes in women with cardiovascular disease: evolving trends over 10 years in the ESC Registry Of Pregnancy And Cardiac disease (ROPAC). Eur Heart J 2019. [Epub ahead of print].

11. Ruys TP, Roos-Hesselink JW, Hall R, et al. Heart failure in pregnant women with cardiac disease: data from the ROPAC. Heart 2014;100:231-8.

12. Galie N, Humbert M, Vachiery JL, et al. 2015 ESC/ERS Guidelines for the diagnosis and treatment of pulmonary hypertension: The Joint Task Force for the Diagnosis and Treatment of Pulmonary Hypertension of the European Society of Cardiology (ESC) and the European Respiratory Society (ERS): Endorsed by: Association for European Paediatric and Congenital Cardiology (AEPC), International Society for Heart and Lung Transplantation (ISHLT). Eur Heart J 2016;37:67-119.

13. Apitz C, Hansmann G, Schranz D. Hemodynamic assessment and acute pulmonary vasoreactivity testing in the evaluation of children with pulmonary vascular disease. Expert consensus statement on the diagnosis and treatment of paediatric pulmonary hypertension. The European Paediatric Pulmonary Vascular Disease Network, endorsed by ISHLT and DGPK. Heart 2016;102 Suppl 2:ii23-9.

14. Kozlik-Feldmann R, Hansmann G, Bonnet D, et al. Pulmonary hypertension in children with congenital heart disease (PAH-CHD, PPHVD-CHD). Expert consensus statement on the diagnosis and treatment of paediatric pulmonary hypertension. The European Paediatric Pulmonary Vascular Disease Network, endorsed by ISHLT and DGPK. Heart 2016;102 Suppl 2:ii42-8.

15. Sliwa K, van Hagen IM, Budts $W$, et al. Pulmonary hypertension and pregnancy outcomes: data from the Registry Of Pregnancy and Cardiac Disease (ROPAC) of the European Society of Cardiology. Eur J Heart Fail 2016;18:1119-28.

16. Nishimura RA, Otto CM, Bonow RO, et al. 2017 AHA/ ACC Focused Update of the 2014 AHA/ACC Guideline for the Management of Patients With Valvular Heart Disease: A Report of the American College of Cardiology/ American Heart Association Task Force on Clinical Practice Guidelines. J Am Coll Cardiol 2017;70:252-89. 
17. Lesniak-Sobelga A, Tracz W, KostKiewicz M, et al. Clinical and echocardiographic assessment of pregnant women with valvular heart diseases--maternal and fetal outcome. Int J Cardiol 2004;94:15-23.

18. Silversides CK, Colman JM, Sermer M, et al. Early and intermediate-term outcomes of pregnancy with congenital aortic stenosis. Am J Cardiol 2003;91:1386-9.

19. Hernandez-Madrid A, Paul T, Abrams D, et al. Arrhythmias in congenital heart disease: a position paper of the European Heart Rhythm Association (EHRA), Association for European Paediatric and Congenital Cardiology (AEPC), and the European Society of Cardiology (ESC) Working Group on Grown-up Congenital heart disease, endorsed by HRS, PACES, APHRS, and SOLAECE. Europace. 2018;20:1719-53.

20. Wasmer K, Eckardt L. Management of supraventricular arrhythmias in adults with congenital heart disease. Heart 2016;102:1614-9.

21. Cuypers JA, Eindhoven JA, Slager MA, et al. The natural and unnatural history of the Mustard procedure: long-term outcome up to 40 years. Eur Heart J 2014;35:1666-74.

22. Couperus LE, Vliegen HW, Zandstra TE, et al. Longterm outcome after atrial correction for transposition of the great arteries. Heart 2019;105:790-6.

Cite this article as: Zengin E, Mueller G, Blankenberg S, von Kodolitsch Y, Rickers C, Sinning C. Pregnancy in adults with congenital heart disease. Cardiovasc Diagn Ther 2019;9(Suppl 2):S416-S423. doi: 10.21037/cdt.2019.07.01
23. Trigas V, Nagdyman N, Pildner von Steinburg S, et al. Pregnancy-related obstetric and cardiologic problems in women after atrial switch operation for transposition of the great arteries. Circ J 2014;78:443-9.

24. O'Byrne ML, Glatz AC, Song L, et al. Association Between Variation in Preoperative Care Before Arterial Switch Operation and Outcomes in Patients With Transposition of the Great Arteries. Circulation 2018;138:2119-29.

25. Lo Rito M, Fittipaldi M, Haththotuwa R, et al. Long-term fate of the aortic valve after an arterial switch operation. J Thorac Cardiovasc Surg 2015;149:1089-94.

26. Tobler D, Williams WG, Jegatheeswaran A, et al. Cardiac outcomes in young adult survivors of the arterial switch operation for transposition of the great arteries. J Am Coll Cardiol 2010;56:58-64.

27. Gewillig M, Brown SC. The Fontan circulation after 45 years: update in physiology. Heart 2016;102:1081-6.

28. Pundi KN, Johnson JN, Dearani JA, et al. 40-Year FollowUp After the Fontan Operation: Long-Term Outcomes of 1,052 Patients. J Am Coll Cardiol 2015;66:1700-10.

29. Khairy P, Fernandes SM, Mayer JE Jr, et al. Long-term survival, modes of death, and predictors of mortality in patients with Fontan surgery. Circulation 2008;117:85-92. 